\title{
Outlier-Resistant Remote State Estimation for Recurrent Neural Networks with Mixed Time-Delays
}

\author{
Jiahui Li, Zidong Wang, Hongli Dong and Gheorghita Ghinea
}

\begin{abstract}
In this paper, a new outlier-resistant state estimation problem is addressed for a class of recurrent neural networks (RNNs) with mixed time-delays. The mixed time-delays comprise both discrete and distributed delays that occur frequently in signal transmissions among artificial neurons. Measurement outputs are sometimes subject to abnormal disturbances (resulting probably from sensor aging/outages/faults/failures and unpredictable environmental changes) leading to measurement outliers that would deteriorate the estimation performance if directly taken into the innovation in the estimator design. We propose to use a certain confidence-dependent saturation function to mitigate the side-effects from the measurement outliers on the estimation error dynamics (EED). Through using a combination of Lyapunov-Krasovskii functional and inequality manipulations, a delay-dependent criterion is established for the existence of the outlier-resistant state estimator ensuring that the corresponding EED achieves the asymptotic stability with a prescribed $H_{\infty}$ performance index. Then, the explicit characterization of the estimator gain is obtained by solving a convex optimization problem. Finally, numerical simulation is carried out to demonstrate the usefulness of the derived theoretical results.
\end{abstract}

Index Terms-Recurrent neural networks; outlier-resistant state estimation; $H_{\infty}$ performance constraint; measurement outliers; mixed timedelays.

\section{INTRODUCTION}

In the past several decades, artificial neural networks (ANNs) have been gaining an ever-increasing popularity in various subject areas such as computer science, brain science as well as cognitive science. According to the ways of connections between neurons and the directions of signal flows, ANNs are roughly divided into two categories, i.e., feed-forward neural networks (FNNs) and recurrent neural networks (RNNs). In FNNs, only the forward transmission of signals is implemented, which means that the output cannot be returned to regulate the input for the purpose of establishing dynamic relationship. On this account, most FNN models are actually learning systems with successful applications in various domains such as pattern recognition, clustering and classification, and adaptive control [7], [8], [35]. Compared with FNNs, a distinguishing feature with RNNs is that certain information feedback occurs between the input and output layers, thereby forming the dynamic relationship. So far, much research attention has devoted to the dynamics analysis issues for RNNs with examples including synchronization [1], [11], stability [22], state estimation [33], [40], [41], [43], and dissipativity [23], [33], and many available RNN-based algorithms have proven to be powerful in a variety of practical applications such as associative

This work was supported in part by the National Natural Science Foundation of China under Grants 61933007, 61873148 and 61873058, the Natural Science Foundation of Heilongiiang Province of China under Grant ZD2019F001, the Key Laboratory of Advanced Perception and Intelligent Control of Highend Equipment of Ministry of Education in Anhui Polytechnic University of China under Grant GDSC202016, and the Alexander von Humboldt Foundation of Germany. (Corresponding author: Hongli Dong.)

$\mathrm{J}$. Li and H. Dong are with the Institute of Complex Systems and Advanced Control, Northeast Petroleum University, Daqing 163318, China. They are also with the Heilongjiang Provincial Key Laboratory of Networking and Intelligent Control, Northeast Petroleum University, Daqing 163318, China. (Email: shiningdhlevip.126.com)

Z. Wang and G. Ghinea are with the Department of Computer Science, Brunel University London, Uxbridge, Middlesex, UB8 3PH, United Kingdom. (Email: Zidong.Wang@brunel.ac.uk) memory, image processing and optimization calculation [18], [21], [32], [38].

Time-delay is a well-known feature with RNNs that often occurs in signal transmission among neurons due primarily to limited communication time of neurons and finite switching speed of amplifiers. It is generally acknowledged that time-delays, if not appropriately dealt with, could cause undesirable dynamical behaviors such as oscillation or even instability. Over the past decade, the discrete time-delays in RNNs have stirred much research attention and a great deal of literature has been available, see e.g. [46], [47] on the synchronization problem, [51] on the boundedness/stability analysis, and [10] on the global stability issue. On the other hand, owing to the usually large number of parallel pathways, RNNs would possess both the temporal and spatial characteristics, and there might be a distribution of conduction velocities along these paths as well as a distribution of propagation delays over a period of time, which gives rise to the so-called distributed time-delays, see e.g. [19], [28], [43]. Clearly, it makes practical sense to look into the impact from mixed (i.e. discrete and distributed) time-delays on RNNs' dynamical behaviors.

For applications of RNNs in achieving certain tasks such as realtime target tracking and navigation, it is often imperative to acquire the full states of all neurons of the RNNs but, unfortunately, this turns out to be a rather difficult mission for a variety of reasons including the large size of the RNNs, the physical constraints of devices and the limited resources with the measurements [48], [49]. Accordingly, the state estimation problem for RNNs has become a focus of research that attracts considerable attention leading to a great number of results reported in the literature, see e.g. [12], [19], [20], [25], [37], [39], [50]. In a wider context, the state estimation strategies for general dynamical systems can be categorized according to the performance indices on the estimation error dynamics such as guaranteed $H_{\infty}$ disturbance rejection/attenuation level, the minimized variance constraints and the set-valued requirements, and the resulting categories are generally $H_{\infty}$ estimation [17], [20], [25], [27], Kalman-type estimation [4], [26], $\mathrm{H}_{2}$ estimation [9], [31], set-valued estimation [34] and set-membership estimation techniques [5], [42], [45].

RNNs have recently become extremely fashionable especially when applied to the deep learning areas such as speech recognition and machine translation. Due to the large scale of the RNNs and the heavy burden of the computation, the implementation (e.g. training and prediction) of the RNNs can be carried out on one site, and the monitoring (e.g. state estimation) can be conducted on another site, where the two sites are connected through networks. Such a scenario of remote state estimation (RSE) of RNNs is particularly realistic in nowadays data-intensive networked environments [29], [30]. In this scenario, the measurement outputs of the RNNs are transmitted to the remote estimator through a communication channel (often wireless or distributed networks) of limited bandwidth that might result in network-induced phenomena (NIP) jeopardizing the information transmission. So far, in the context of RSE for RNNs, some wellknown NIP have been thoroughly investigated, see e.g. [25] for missing measurements, [19] for fading channels, [37] for unreliable links, and [29] for communication bandwidth constraints. In [30], the Round-Robin protocol has been introduced to actively mitigate the NIP's negative impact on the neuron state estimation problem. 


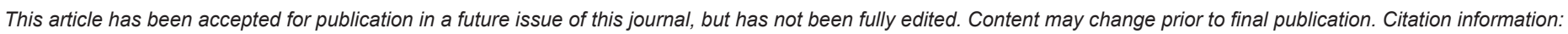
DOI10.1109/TNNLS.2020.2991151, IEEE Transactions on Neural Networks and Learning Systems

For large-scale RNNs, the measurement outputs are sometimes subject to abnormal disturbances resulting probably from 1) sensor aging/outages/faults/failures; 2) unpredictable/sudden/dramatic environmental changes; and 3) cyber-attack from adversaries. Such kind of abnormal outputs are referred to as measurement outliers which, if not appropriately tackled, would seriously deteriorate the estimation accuracy for the RSE problems for RNNs. To be more specific, if the measurement outliers are directly utilized in estimating the states of the artificial neurons, the corresponding innovation value would become abnormal, thereby leading to the undesirable state estimates that are out of range. Up to now, in contrast to the rich literature regarding various NIP, the available results on the RSE problems for RNNs subject to measurement outliers have been very few, and this constitutes the main motivation of our current investigation.

It should be noticed that the measurement outlier problem has received some initial research attention in the general area of state estimation or filtering. For example, in [15], a new type of Kalman filter has been constructed which is insensitive to measurement outliers. Moreover, a modified maximum likelihood estimator has been designed in [2] which is robust to the possible outliers. Recently, a novel observer has been constructed in [3] with a saturated output injection to attenuate the impact of measurement outliers on the state estimation problems. Unfortunately, when it comes to the RSE problems for RNNs, the relevant literature has been very little, not to mention the case when the $H_{\infty}$ disturbance attention/rejection requirement is also a concern. It is, therefore, our main purpose of this paper to deal with the outlier-resistant RSE problem by employing a certain confidence-dependent saturation function to mitigate the side-effects from the measurement outliers on the estimation error dynamics (EED).

Inspired by the above discussions, the main aim of this work is to look into the outlier-resistant RSE problem for a class of RNNs subject to mixed time-delays. This appears to be a rather challenging research topic involving two substantial objectives as follows: 1) the design of an effective estimator to mitigate the effects of measurement outliers on the RSE issue; and 2) the determination of the estimator parameters such that the EED is asymptotically stable with a specified $H_{\infty}$ performance index. Accordingly, the main contribution of our investigation is outlined as threefold: 1) a new outlier-resistant state estimation problem is, for the first time, put forward for a class of RNNs subject to mixed time-delays; 2) a confidence-dependent saturation function is introduced into the estimator structure so as to attenuate the side-effects from the measurement outliers on the EED; and 3) a delay-dependent criterion is derived to guarantee the asymptotic stability of the corresponding EED with guaranteed $H_{\infty}$ performance constraint.

Notation: The notation utilized here is quite normative except where otherwise declared. $\mathbb{R}^{n}$ represents the $n$-dimensional Euclidean space and $\mathbb{R}^{n \times m}$ is the set of all real matrices of dimension $n \times m . \mathbb{Z}^{-}$ denotes the set of all negative integers. The occurrence probability of the event "." is denoted by Prob $\{\cdot\} . \mathbb{E}\{x\}$ means the expectation of a random variable $x$. For a vector $y,\|y\|$ stands for its Euclidean norm. The symbol $\star$ indicates an ellipsis for symmetry-induced terms. For a square matrix $M, \lambda_{\max }(M)$ means the maximum eigenvalue of $M$. $l_{2}[0, \infty)$ denotes the space of square-integrable vector functions over $[0, \infty)$.

\section{Problem Formulation And Preliminaries}

Consider the following $n$-neuron neural networks with mixed timedelays:

$$
\left\{\begin{aligned}
x_{k+1} & =E x_{k}+D \bar{g}\left(x_{k-d_{k}}\right)+B \sum_{\tau=1}^{\infty} \mu_{\tau} \bar{f}\left(x_{k-\tau}\right)+H w_{k} \\
y_{k} & =M x_{k} \\
x_{s} & =\varphi_{s}, s \in \mathbb{Z}^{-}
\end{aligned}\right.
$$

where $x_{k} \in \mathbb{R}^{n}$ represents the neural state vector with the initial conditions $x_{s}=\varphi_{s}, s \in \mathbb{Z}^{-} ; \bar{g}\left(x_{k}\right) \in \mathbb{R}^{n}$ and $\bar{f}\left(x_{k}\right) \in \mathbb{R}^{n}$ denote the nonlinear activation functions; $w_{k} \in \mathbb{R}^{n}$ is the disturbance input belonging to $l_{2}[0, \infty) ; y_{k} \in \mathbb{R}^{m}$ is the output of the neural networks; $E \triangleq \operatorname{diag}\left\{e_{1}, e_{2}, \ldots, e_{n}\right\}$ is a real constant diagonal matrix; $D \triangleq$ $\left(d_{i j}\right)_{n \times n}$ is the time-varying delayed connection weight matrix and $B \triangleq\left(b_{i j}\right)_{n \times n}$ denotes the distributively delayed connection weight matrix; $d_{k}$ is a positive integer representing the discrete time-varying delays that satisfy $\underline{d} \leq d_{k} \leq \bar{d}$, in which $\underline{d}$ and $\bar{d}$ are constant positive integers denoting, respectively, the lower and upper bounds of $d_{k} ; \tau$ describes the infinitely distributed delays; and $M$ and $H$ are known constant matrices with compatible dimensions.

Assumption 1: The constants $\mu_{\tau} \geq 0(\tau=1,2, \ldots, \infty)$ satisfy the following convergence condition:

$$
\bar{\mu}=\sum_{\tau=1}^{\infty} \mu_{\tau}<\infty \text { and } \bar{\mu}_{0}=\sum_{\tau=1}^{\infty} \tau \mu_{\tau}<\infty .
$$

Assumption 2: The nonlinear activation functions $\bar{g}(\cdot)$ and $\bar{f}(\cdot)$ are continuous which satisfy $\bar{g}(0)=0, \bar{f}(0)=0$ and the following sector-bounded constraints:

$$
\begin{gathered}
\left(\bar{g}(x)-\bar{g}(y)-L_{1}(x-y)\right)^{T}\left(\bar{g}(x)-\bar{g}(y)-L_{2}(x-y)\right) \leq 0, \\
\left(\bar{f}(x)-\bar{f}(y)-T_{1}(x-y)\right)^{T}\left(\bar{f}(x)-\bar{f}(y)-T_{1}(x-y)\right) \leq 0
\end{gathered}
$$

for all $x, y \in \mathbb{R}^{n}$, where $L_{1}, L_{2}, T_{1}$ and $T_{2}$ are real-valued matrices with appropriate dimensions.

The objective of this paper is to design an effective estimator for estimating the neurons' states $\hat{x}_{k}$ through available measurement outputs $y_{k}$. As discussed in the introduction, measurement outliers do exist which, if not adequately handled, would deteriorate the estimator performance or even make the error dynamics unstable. To resolve such a problem, a so-called outlier-resistant estimator is constructed as follows:

$$
\left\{\begin{aligned}
\hat{x}_{k+1}= & E \hat{x}_{k}+D \bar{g}\left(\hat{x}_{k-d_{k}}\right)+B \sum_{\tau=1}^{\infty} \mu_{\tau} \bar{f}\left(\hat{x}_{k-\tau}\right) \\
& +K \sigma\left(y_{k}-M \hat{x}_{k}\right) \\
\hat{x}_{s}= & 0, s \in \mathbb{Z}^{-}
\end{aligned}\right.
$$

where $\hat{x}_{k}$ and $\hat{x}_{s}$ are, respectively, the estimates of $x_{k}$ and $x_{s}$, and $K$ is the estimator gain to be designed. In particular, $\sigma(\cdot): \mathbb{R}^{m} \mapsto \mathbb{R}^{m}$ is a saturation function defined as:

$$
\sigma(z) \triangleq\left[\begin{array}{llll}
\sigma_{1}^{T}\left(z_{1}\right) & \sigma_{2}^{T}\left(z_{2}\right) & \cdots & \sigma_{m}^{T}\left(z_{m}\right)
\end{array}\right]^{T}
$$

in which $\sigma_{\iota}\left(z_{\iota}\right) \triangleq \operatorname{sign}\left(z_{\iota}\right) \min \left\{z_{\iota, \max },\left|z_{\iota}\right|\right\}(\iota=1,2, \ldots, m)$ with $z_{\iota, \max }$ being the $\iota$ th element of $z_{\max }$ (i.e., the saturation level).

Remark 1: The outliers are understood as a small portion of data that deviates severely from the trend of most data, and the outliers are often regarded as "bad values" by data analysts. Outliers might result from equipment failures, execution errors, cyber-attacks and so on. Clearly, for our addressed RSE problem, the state estimator to be designed should be insensitive to the outlier where such insensitivity should be quantified. As such, the main objective of this paper is 
to establish an effective estimation scheme to weaken the influence resulting from the measurement outliers.

Remark 2: In the proposed estimator (4), we have purposely introduced a saturation function $\sigma(\cdot): \mathbb{R}^{m} \mapsto \mathbb{R}^{m}$ so as to specify a certain bound on the innovation $y_{k}-M \hat{x}_{k}$. The reason for doing this is that, if $y_{k}$ is indeed an outlier that is directly used to calculate the innovation, then the produced state estimate $\hat{x}_{k+1}$ could differ significantly from the estimates at other time points, which means the resulting $\hat{x}_{k+1}$ might be unreliable and even lead to the divergence of the error dynamics. With the introduced saturation function $\sigma(\cdot)$, the innovation value would have specified bounds, thereby alleviating the risk of unwanted abnormality/divergence. Here, the saturation level $z_{\iota, \max }$ can be determined a priori according to engineering practice, that is, our confidence/knowledge on the measurement outputs. In this sense, the saturation function $\sigma(\cdot)$ is said to be confidence-dependent and the estimator (4) is called outlier-resistant. Note that, when the saturation level goes to infinite, that is, there is no proposed limit on the innovation, the estimator (4) will be degenerated into the traditional one.

Letting $e_{k} \triangleq x_{k}-\hat{x}_{k}, g\left(e_{k}\right) \triangleq \bar{g}\left(x_{k}\right)-\bar{g}\left(\hat{x}_{k}\right)$ and $f\left(e_{k}\right) \triangleq$ $\bar{f}\left(x_{k}\right)-\bar{f}\left(\hat{x}_{k}\right)$, the EED is obtained from (1) and (4) as follows:

$$
\begin{aligned}
e_{k+1}= & E e_{k}+D g\left(e_{k-d_{k}}\right)+B \sum_{\tau=1}^{\infty} \mu_{\tau} f\left(e_{k-\tau}\right) \\
& +H w_{k}-K \sigma\left(M e_{k}\right) .
\end{aligned}
$$

The main objective of this paper is to develop an outlier-resistant RSE scheme such that the following requirements are met simultaneously:

a) for $w_{k}=0$, the existence condition is acquired for the desired estimator (4) ensuring the asymptotic stability of the EED $e_{k}$;

b) under the zero-initial conditions, for a given disturbance attenuation level $\hbar>0$ and all nonzero $w_{k}$, the EED $e_{k}$ from (5) satisfies the following $H_{\infty}$ performance constraint:

$$
\sum_{k=0}^{\infty}\left\|e_{k}\right\|^{2} \leq \hbar^{2} \sum_{k=0}^{\infty}\left\|w_{k}\right\|^{2}
$$

\section{MAIN RESULTS}

Lemma 1: [44] Assume that the saturation function $\sigma(\cdot)$ belongs to $\left[U_{1}, U_{2}\right]$ with some given diagonal matrices $U_{1}, U_{2}$, where $U_{1} \geq 0$, $U_{2} \geq 0$ and $U_{2} \geq U_{1} . \sigma(\cdot)$ satisfies the following inequality:

$$
\left[\sigma\left(M e_{k}\right)-U_{1} M e_{k}\right]^{T}\left[\sigma\left(M e_{k}\right)-U_{2} M e_{k}\right] \leq 0 .
$$

By (7), the nonlinear function $\sigma\left(M e_{k}\right)$ can be divided into a linear and a nonlinear part

$$
\sigma\left(M e_{k}\right)=U_{1} M e_{k}+\psi\left(M e_{k}\right)
$$

and the nonlinear part $\psi\left(M e_{k}\right)$ satisfies $\psi\left(M e_{k}\right) \in \boldsymbol{\Psi}$, where $\boldsymbol{\Psi}$ is described as

$$
\Psi \triangleq\left\{\psi: \psi^{T}\left(M e_{k}\right)\left[\psi\left(M e_{k}\right)-U M e_{k}\right] \leq 0\right\}, U \triangleq U_{2}-U_{1} .
$$

Lemma 2: [36] Let $S \in \mathbb{R}^{n \times n}$ be a positive semi-definite matrix, $x_{i} \in \mathbb{R}^{n}$ be a vector and $\mu_{i} \geq 0(i=1,2, \ldots, \infty)$ be a nonnegative scalar. If the series concerned are convergent, the following inequality is true:

$$
\left(\sum_{i=1}^{+\infty} \mu_{i} x_{i}\right)^{T} S\left(\sum_{i=1}^{+\infty} \mu_{i} x_{i}\right) \leq\left(\sum_{i=1}^{+\infty} \mu_{i}\right)\left(\sum_{i=1}^{+\infty} \mu_{i} x_{i}^{T} S x_{i}\right) .
$$

Lemma 3: [6] Given constant matrices $\Gamma_{1}, \Gamma_{2}, \Gamma_{3}$ where $\Gamma_{1}=$ $\Gamma_{1}^{T}$ and $\Gamma_{2}=\Gamma_{2}^{T}>0$, then $\Gamma_{1}+\Gamma_{3}^{T} \Gamma_{2}^{-1} \Gamma_{3}<0$ if and only if

$$
\left[\begin{array}{cc}
\Gamma_{1} & \Gamma_{3}^{T} \\
\Gamma_{3} & -\Gamma_{2}
\end{array}\right]<0 \text { or }\left[\begin{array}{cc}
-\Gamma_{2} & \Gamma_{3} \\
\Gamma_{3}^{T} & \Gamma_{1}
\end{array}\right]<0
$$

Now, we are ready to present a delay-dependent criterion for the existence of the desired outlier-resistant estimator (4) for ensuring the asymptotic stability of (5).

Theorem 1: Consider the delayed neural networks (1) and let the estimator gain $K$ be given. The corresponding EED (5) is asymptotically stable if there exist positive definite matrices $P, R$ and $Z$, and positive constant scalars $\lambda_{1}$ and $\lambda_{2}$ satisfying

$$
\Pi=\left[\begin{array}{cccccc}
\Pi_{11} & \Pi_{12} & \Pi_{13} & \Pi_{14} & \Pi_{15} & \Pi_{16} \\
\star & \Pi_{22} & 0 & 0 & 0 & 0 \\
\star & \star & \Pi_{33} & 0 & \Pi_{35} & \Pi_{36} \\
\star & \star & \star & \Pi_{44} & 0 & 0 \\
\star & \star & \star & \star & \Pi_{55} & \Pi_{56} \\
\star & \star & \star & \star & \star & \Pi_{66}
\end{array}\right]<0
$$

where

$$
\begin{aligned}
& \Pi_{11} \triangleq \bar{E}^{T} P \bar{E}-P-\lambda_{1} \tilde{L}_{1}-\lambda_{2} \tilde{T}_{1}, \quad \Pi_{12} \triangleq \lambda_{1} \tilde{L}_{2}, \\
& \Pi_{13} \triangleq \bar{E}^{T} P D, \quad \Pi_{14} \triangleq \lambda_{2} \tilde{T}_{2}, \quad \Pi_{15} \triangleq \bar{E}^{T} P B, \\
& \Pi_{16} \triangleq-\bar{E}^{T} P K+\frac{1}{2} M^{T} U^{T}, \quad \Pi_{22} \triangleq(1+\bar{d}-\underline{d}) R-\lambda_{1} I, \\
& \Pi_{33} \triangleq D^{T} P D-R, \quad \Pi_{35} \triangleq D^{T} P B, \quad \Pi_{36} \triangleq-D^{T} P K, \\
& \Pi_{44} \triangleq \bar{\mu} Z-\lambda_{2} I, \quad \Pi_{55} \triangleq B^{T} P B-\frac{1}{\bar{\mu}} Z, \quad \Pi_{56} \triangleq-B^{T} P K, \\
& \Pi_{66} \triangleq K^{T} P K-I, \quad \bar{E} \triangleq E-K U_{1} M, \quad \tilde{L}_{1} \triangleq \frac{L_{1}^{T} L_{2}+L_{2}^{T} L_{1}}{2}, \\
& \tilde{L}_{2} \triangleq \frac{\left(L_{1}+L_{2}\right)^{T}}{2}, \quad \tilde{T}_{1} \triangleq \frac{T_{1}^{T} T_{2}+T_{2}^{T} T_{1}}{2}, \quad \tilde{T}_{2} \triangleq \frac{\left(T_{1}+T_{2}\right)^{T}}{2} .
\end{aligned}
$$

Proof: In order to analyze the stability of the EED (5) with $w_{k}=0$, we select the following Lyapunov-Krasovskii functional:

$$
V\left(e_{k}\right)=V_{1}\left(e_{k}\right)+V_{2}\left(e_{k}\right)+V_{3}\left(e_{k}\right)
$$

where

$$
\begin{aligned}
V_{1}\left(e_{k}\right) \triangleq & e_{k}^{T} P e_{k}, \\
V_{2}\left(e_{k}\right) \triangleq & \sum_{j=k-d_{k}}^{k-1} g^{T}\left(e_{j}\right) R g\left(e_{j}\right) \\
& +\sum_{q=k-\bar{d}+1}^{k-\underline{d}} \sum_{j=q}^{k-1} g^{T}\left(e_{j}\right) R g\left(e_{j}\right), \\
V_{3}\left(e_{k}\right) \triangleq & \sum_{\tau=1}^{\infty} \mu_{\tau} \sum_{j=k-\tau}^{k-1} f^{T}\left(e_{j}\right) Z f\left(e_{j}\right) .
\end{aligned}
$$

Along the trajectories of system (5), we calculate the difference of $V\left(e_{k}\right)$ as follows:

$$
\Delta V\left(e_{k}\right)=\Delta V_{1}\left(e_{k}\right)+\Delta V_{2}\left(e_{k}\right)+\Delta V_{3}\left(e_{k}\right)
$$

where

$$
\begin{aligned}
\Delta V_{1}\left(e_{k}\right)= & V_{1}\left(e_{k+1}\right)-V_{1}\left(e_{k}\right) \\
= & {\left[E e_{k}+D g\left(e_{k-d_{k}}\right)+B \sum_{\tau=1}^{\infty} \mu_{\tau} f\left(e_{k-\tau}\right)\right.} \\
& \left.-K \sigma\left(M e_{k}\right)\right]^{T} P\left[E e_{k}+D g\left(e_{k-d_{k}}\right)\right. \\
& \left.+B \sum_{\tau=1}^{\infty} \mu_{\tau} f\left(e_{k-\tau}\right)-K \sigma\left(M e_{k}\right)\right]-e_{k}^{T} P e_{k}
\end{aligned}
$$




$$
\begin{aligned}
& \Delta V_{2}\left(e_{k}\right)=V_{2}\left(e_{k+1}\right)-V_{2}\left(e_{k}\right) \\
& =\sum_{j=k+1-d_{k+1}}^{k} g^{T}\left(e_{j}\right) R g\left(e_{j}\right)+\sum_{q=k-\bar{d}+2}^{k+1-\underline{d}} \sum_{j=q}^{k} g^{T}\left(e_{j}\right) R g\left(e_{j}\right) \\
& -\sum_{j=k-d_{k}}^{k-1} g^{T}\left(e_{j}\right) R g\left(e_{j}\right)-\sum_{q=k-\bar{d}+1}^{k-\underline{d}} \sum_{j=q}^{k-1} g^{T}\left(e_{j}\right) R g\left(e_{j}\right) \\
& =\left(g^{T}\left(e_{k}\right) R g\left(e_{k}\right)+\sum_{j=k+1-d_{k+1}}^{k-1} g^{T}\left(e_{j}\right) R g\left(e_{j}\right)\right. \\
& \left.-\sum_{j=k+1-d_{k+1}}^{k-1} g^{T}\left(e_{j}\right) R g\left(e_{j}\right)-g^{T}\left(e_{k-d_{k}}\right) R g\left(e_{k-d k}\right)\right) \\
& +\left(\sum_{j=k+1-\underline{d}}^{k-1} g^{T}\left(e_{j}\right) R g\left(e_{j}\right)-\sum_{j=k+1-\bar{d}}^{k-1} g^{T}\left(e_{j}\right) R g\left(e_{j}\right)\right. \\
& \left.+(\bar{d}-\underline{d}) g^{T}\left(e_{k}\right) R g\left(e_{k}\right)\right) \\
& =(1+\bar{d}-\underline{d}) g^{T}\left(e_{k}\right) R g\left(e_{k}\right)-g^{T}\left(e_{k-d_{k}}\right) R g\left(e_{k-d_{k}}\right) \\
& -\sum_{j=k+1-\bar{d}}^{k-\underline{d}} g^{T}\left(e_{j}\right) R g\left(e_{j}\right) \\
& \leq(1+\bar{d}-\underline{d}) g^{T}\left(e_{k}\right) R g\left(e_{k}\right)-g^{T}\left(e_{k-d_{k}}\right) R g\left(e_{k-d_{k}}\right)
\end{aligned}
$$

and

$$
\begin{aligned}
\Delta V_{3}\left(e_{k}\right)= & V_{3}\left(e_{k+1}\right)-V_{3}\left(e_{k}\right) \\
= & \sum_{\tau=1}^{\infty} \mu_{\tau} \sum_{j=k-\tau+1}^{k} f^{T}\left(e_{j}\right) Z f\left(e_{j}\right) \\
& -\sum_{\tau=1}^{\infty} \mu_{\tau} \sum_{j=k-\tau}^{k-1} f^{T}\left(e_{j}\right) Z f\left(e_{j}\right) \\
= & \sum_{\tau=1}^{\infty} \mu_{\tau} f^{T}\left(e_{k}\right) Z f\left(e_{k}\right)-\sum_{\tau=1}^{\infty} \mu_{\tau} f^{T}\left(e_{k-\tau}\right) Z f\left(e_{k-\tau}\right) \\
= & \bar{\mu} f^{T}\left(e_{k}\right) Z f\left(e_{k}\right)-\sum_{\tau=1}^{\infty} \mu_{\tau} f^{T}\left(e_{k-\tau}\right) Z f\left(e_{k-\tau}\right) . \quad(14)
\end{aligned}
$$

Applying Lemma 2 to (14), one has

$$
\begin{aligned}
\Delta V_{3}\left(e_{k}\right) \leq & \bar{\mu} f^{T}\left(e_{k}\right) Z f\left(e_{k}\right)-\frac{1}{\bar{\mu}}\left(\sum_{\tau=1}^{\infty} \mu_{\tau} f^{T}\left(e_{k-\tau}\right)\right) \\
& \times Z\left(\sum_{\tau=1}^{\infty} \mu_{\tau} f\left(e_{k-\tau}\right)\right) .
\end{aligned}
$$

According to Assumption 2, one observes from (3) that

$$
\begin{aligned}
& -\lambda_{1} g^{T}\left(e_{k}\right) g\left(e_{k}\right)-\lambda_{1} e_{k}^{T} \tilde{L}_{1} e_{k}+2 \lambda_{1} e_{k}^{T} \tilde{L}_{2} g\left(e_{k}\right) \geq 0, \\
& -\lambda_{2} f^{T}\left(e_{k}\right) f\left(e_{k}\right)-\lambda_{2} e_{k}^{T} \tilde{T}_{1} e_{k}+2 \lambda_{2} e_{k}^{T} \tilde{T}_{2} f\left(e_{k}\right) \geq 0 .
\end{aligned}
$$

Taking (8)-(9) and (12)-(16) into consideration, we arrive at

$$
\begin{aligned}
\Delta V\left(e_{k}\right) \leq & e_{k}^{T} \bar{E}^{T} P \bar{E} e_{k}+2 e_{k}^{T} \bar{E}^{T} P D g\left(e_{k-d_{k}}\right) \\
& +2 e_{k}^{T} \bar{E}^{T} P B \sum_{\tau=1}^{\infty} \mu_{\tau} f\left(e_{k-\tau}\right) \\
& -2 e_{k}^{T} \bar{E}^{T} P K \psi\left(M e_{k}\right) \\
& +g^{T}\left(e_{k-d_{k}}\right) D^{T} P D g\left(e_{k-d_{k}}\right) \\
& +2 g^{T}\left(e_{k-d_{k}}\right) D^{T} P B \sum_{\tau=1}^{\infty} \mu_{\tau} f\left(e_{k-\tau}\right) \\
& -2 g^{T}\left(e_{k-d_{k}}\right) D^{T} P K \psi\left(M e_{k}\right)
\end{aligned}
$$

$$
\begin{aligned}
& +\sum_{\tau=1}^{\infty} \mu_{\tau} f^{T}\left(e_{k-\tau}\right) B^{T} P B \sum_{\tau=1}^{\infty} \mu_{\tau} f\left(e_{k-\tau}\right) \\
& -2 \sum_{\tau=1}^{\infty} \mu_{\tau} f^{T}\left(e_{k-\tau}\right) B^{T} P K \psi\left(M e_{k}\right) \\
& +\psi^{T}\left(M e_{k}\right) K^{T} P K \psi\left(M e_{k}\right) \\
& +(1+\bar{d}-\underline{d}) g^{T}\left(e_{k}\right) R g\left(e_{k}\right)-g^{T}\left(e_{k-d_{k}}\right) \\
& \times R g\left(e_{k-d_{k}}\right)-e_{k}^{T} P e_{k}+\bar{\mu} f^{T}\left(e_{k}\right) Z f\left(e_{k}\right) \\
& -\frac{1}{\bar{\mu}}\left(\sum_{\tau=1}^{\infty} \mu_{\tau} f^{T}\left(e_{k-\tau}\right)\right) Z\left(\sum_{\tau=1}^{\infty} \mu_{\tau} f\left(e_{k-\tau}\right)\right) \\
& -\lambda_{1} g^{T}\left(e_{k}\right) g\left(e_{k}\right)-\lambda_{1} e_{k}^{T} \tilde{L}_{1} e_{k}+2 \lambda_{1} e_{k}^{T} \tilde{L}_{2} g\left(e_{k}\right) \\
& -\lambda_{2} f^{T}\left(e_{k}\right) f\left(e_{k}\right)-\lambda_{2} e_{k}^{T} \tilde{T}_{1} e_{k}+2 \lambda_{2} e_{k}^{T} \tilde{T}_{2} f\left(e_{k}\right) \\
& -\psi^{T}\left(M e_{k}\right)\left[\psi\left(M e_{k}\right)-U M e_{k}\right] \\
& =\xi_{k}^{T} \Pi \xi_{k}
\end{aligned}
$$

where

$$
\begin{aligned}
\xi_{k} & \triangleq\left[\begin{array}{ll}
\xi_{1 k}^{T} & \xi_{2 k}^{T}
\end{array}\right]^{T}, \\
\xi_{1 k} & \triangleq\left[\begin{array}{lll}
e_{k}^{T} & g^{T}\left(e_{k}\right) & g^{T}\left(e_{k-d_{k}}\right)
\end{array}\right]^{T}, \\
\xi_{2 k} & \triangleq\left[\begin{array}{lll}
f^{T}\left(e_{k}\right) & \sum_{\tau=1}^{\infty} \mu_{\tau} f^{T}\left(e_{k-\tau}\right) & \psi^{T}\left(M e_{k}\right)
\end{array}\right]^{T} .
\end{aligned}
$$

Since $\lambda_{\max }(\Pi)<0$, it is obvious to see from (17) that

$$
\triangle V\left(e_{k}\right) \leq \lambda_{\max }(\Pi)\left\|e_{k}\right\|^{2} .
$$

For a given positive integer $N$, summing both sides of (18) from 1 to $N$ with respect to $k$ yields

$$
V\left(e_{N}\right)-V\left(e_{0}\right) \leq \lambda_{\max }(\Pi) \sum_{k=1}^{N}\left\|e_{k}\right\|^{2},
$$

which implies

$$
-\lambda_{\max }(\Pi) \sum_{k=1}^{N}\left\|e_{k}\right\|^{2} \leq V\left(e_{0}\right) .
$$

Letting $N \rightarrow \infty$, we see that the series $\sum_{k=0}^{+\infty}\left\|e_{k}\right\|^{2}$ is convergent, which further implies that the EED (5) is asymptotic stable and the proof is now complete.

In what follows, a delay-dependent criterion will be established for the existence of the desired outlier-resistant state estimator for ensuring the $H_{\infty}$ performance constraint of the EED (5).

Theorem 2: Let the disturbance attenuation level $\hbar>0$ and estimator parameter $K$ be specified. The EED (5) achieves the asymptotic stability with $H_{\infty}$ performance constraint (6) if there exist symmetric positive definite matrices $P>0, R>0$ and $Z>0$ as well as positive scalars $\lambda_{1}$ and $\lambda_{2}$ such that the following condition holds:

$$
\tilde{\Pi}=\left[\begin{array}{ccccccc}
\bar{\Pi}_{11} & \Pi_{12} & \Pi_{13} & \Pi_{14} & \Pi_{15} & \Pi_{16} & \Pi_{17} \\
\star & \Pi_{22} & 0 & 0 & 0 & 0 & 0 \\
\star & \star & \Pi_{33} & 0 & \Pi_{35} & \Pi_{36} & \Pi_{37} \\
\star & \star & \star & \Pi_{44} & 0 & 0 & 0 \\
\star & \star & \star & \star & \Pi_{55} & \Pi_{56} & \Pi_{57} \\
\star & \star & \star & \star & \star & \Pi_{66} & \Pi_{67} \\
\star & \star & \star & \star & \star & \star & \Pi_{77}
\end{array}\right]<0
$$

where

$$
\begin{aligned}
& \bar{\Pi}_{11} \triangleq \Pi_{11}+I, \quad \Pi_{17} \triangleq \bar{E}^{T} P H, \quad \Pi_{37} \triangleq D^{T} P H, \\
& \Pi_{57} \triangleq B^{T} P H, \quad \Pi_{67} \triangleq-K^{T} P H, \quad \Pi_{77} \triangleq H^{T} P H-\hbar^{2} I .
\end{aligned}
$$

Proof: In order to discuss the $H_{\infty}$ disturbance attenuation performance of the estimator, we adopt the same Lyapunov-Krasovskii 


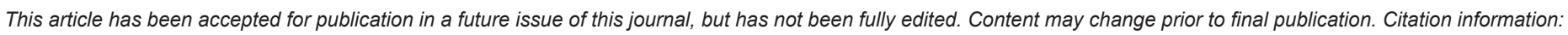
DOI10.1109/TNNLS.2020.2991151, IEEE Transactions on Neural Networks and Learning Systems

functional as that in proof of Theorem 1 with $w_{k} \neq 0$. Furthermore, we assume zero-initial conditions, i.e. $\left\{e_{k}\right\}_{k \in \mathbb{Z}^{-}}=0$ and establish the following cost function:

$$
J_{k}^{*}=\left\|e_{k}\right\|^{2}-\hbar^{2}\left\|w_{k}\right\|^{2} .
$$

By similar treatment as in Theorem 1, it is easily seen that

$$
\Delta V\left(e_{k}\right)+J_{k}^{*} \leq \tilde{\xi}_{k}^{T} \tilde{\Pi} \tilde{\xi}_{k}
$$

where

$$
\tilde{\xi}_{k} \triangleq\left[\begin{array}{ll}
\xi_{k}^{T} & w_{k}^{T}
\end{array}\right]^{T} .
$$

In terms of (19), one has

$$
\Delta V\left(e_{k}\right)<-J_{k}^{*} .
$$

Summing both sides of inequality (21) from 0 to $\infty$ regarding $k$ results in

$$
V\left(e_{\infty}\right)-V\left(e_{0}\right) \leq \sum_{k=0}^{\infty} \hbar^{2}\left\|w_{k}\right\|^{2}-\sum_{k=0}^{\infty}\left\|e_{k}\right\|^{2},
$$

which means

$$
\sum_{k=0}^{\infty}\left\|e_{k}\right\|^{2} \leq \hbar^{2} \sum_{k=0}^{\infty}\left\|w_{k}\right\|^{2} .
$$

The proof is now complete.

In the following, we are in a position to determine the gain matrix of the outlier-resistant state estimator (4).

Theorem 3: Consider the system (1) with mixed time-delays. Assume that there exist symmetric positive definite matrices $P>0$, $R>0$ and $Z>0$, a matrix $X>0$, and positive scalars $\lambda_{1}$ and $\lambda_{2}$ such that the following linear matrix inequality (LMI) is true:

$$
\left[\begin{array}{cc}
\Pi_{0} & \tilde{\Sigma}^{T} \\
\star & -P
\end{array}\right]<0
$$

where

$$
\begin{aligned}
& \Pi_{0} \triangleq\left[\begin{array}{ccccccc}
\tilde{\Pi}_{11} & \Pi_{12} & 0 & \Pi_{14} & 0 & \frac{1}{2} M^{T} U^{T} & 0 \\
\star & \Pi_{22} & 0 & 0 & 0 & 0 & 0 \\
\star & \star & -R & 0 & 0 & 0 & 0 \\
\star & \star & \star & \Pi_{44} & 0 & 0 & 0 \\
\star & \star & \star & \star & -\frac{1}{\bar{\mu}} Z & 0 & 0 \\
\star & \star & \star & \star & \star & -I & 0 \\
\star & \star & \star & \star & \star & \star & -\hbar^{2} I
\end{array}\right] \\
& \tilde{\Sigma} \triangleq\left[\begin{array}{lllllll}
P E-X U_{1} M & 0_{n \times n} & 0_{n \times n} & P D & P B & -X & P H
\end{array}\right], \\
& \tilde{\Pi}_{11} \triangleq-P-\lambda_{1} \tilde{L}_{1}-\lambda_{2} \tilde{T}_{1}+I \text {. }
\end{aligned}
$$

Then, the EED (5) is asymptotically stable while achieving the $H_{\infty}$ performance constraint (6) and the corresponding estimator gain matrix is computed as $K=P^{-1} X$.

Proof: Let us first split $\tilde{\Pi}$ as follows:

$$
\tilde{\Pi}=\Pi_{0}+\Sigma^{T} P \Sigma<0
$$

where

$$
\Sigma \triangleq\left[\begin{array}{lllllll}
E-K U_{1} M & 0_{n \times n} & D & 0_{n \times n} & B & -K & H
\end{array}\right] .
$$

By employing Lemma 3, we deduce from (23) that

$$
\left[\begin{array}{cc}
\Pi_{0} & \Sigma^{T} \\
\star & -P^{-1}
\end{array}\right]<0
$$

Pre- and post-multiplying inequality (24) by $\operatorname{diag}\{I, P\}$, one has

$$
\left[\begin{array}{cc}
\Pi_{0} & \Sigma^{T} P \\
\star & -P
\end{array}\right]<0
$$

Letting $X=P K$, it is easy to see that (25) is equivalent to (22), which completes the proof.
In the case the saturation effects are removed from the estimator (4), the innovation becomes unconstrained and a traditional state estimator is obtained as follows:

$$
\left\{\begin{aligned}
\hat{x}_{k+1}= & E \hat{x}_{k}+D \bar{g}\left(\hat{x}_{k-d_{k}}\right)+B \sum_{\tau=1}^{\infty} \mu_{\tau} \bar{f}\left(\hat{x}_{k-\tau}\right) \\
& +\bar{K}\left(y_{k}-M \hat{x}_{k}\right) \\
\hat{x}_{s}= & 0, s \in \mathbb{Z}^{-} .
\end{aligned}\right.
$$

In this case, the following corollary is easily accessible from Theorem 3.

Corollary 1: Assume that there exist symmetric positive definite matrices $\bar{P}>0, \bar{R}>0$ and $\bar{Z}>0$, a matrix $\bar{X}>0$, and positive scalars $\bar{\lambda}_{1}$ and $\bar{\lambda}_{2}$ such that the following LMI holds:

$$
\left[\begin{array}{cc}
\Omega_{0} & \Omega^{T} \\
\star & -\bar{P}
\end{array}\right]<0
$$

where

$$
\begin{aligned}
& \Omega_{0} \triangleq\left[\begin{array}{cccccc}
\Omega_{11} & \lambda_{1} \tilde{L}_{2} & 0 & \lambda_{2} \tilde{T}_{2} & 0 & 0 \\
\star & \Omega_{22} & 0 & 0 & 0 & 0 \\
\star & \star & -\bar{R} & 0 & 0 & 0 \\
\star & \star & \star & \Omega_{44} & 0 & 0 \\
\star & \star & \star & \star & -\frac{1}{\bar{\mu}} \bar{Z} & 0 \\
\star & \star & \star & \star & \star & -\hbar^{2} I
\end{array}\right] \text {, } \\
& \Omega \triangleq\left[\begin{array}{llllll}
\bar{P} E-\bar{X} M & 0_{n \times n} & \bar{P} D & 0_{n \times n} & \bar{P} B & \bar{P} H
\end{array}\right], \\
& \Omega_{11} \triangleq-\bar{P}-\lambda_{1} \tilde{L}_{1}-\lambda_{2} \tilde{T}_{1}+I, \quad \Omega_{22} \triangleq(1+\bar{d}-\underline{d}) \bar{R}-\bar{\lambda}_{1} I, \\
& \Omega_{44} \triangleq \bar{\mu} \bar{Z}-\bar{\lambda}_{2} I \text {. }
\end{aligned}
$$

Then, the EED (5) of system (1) is asymptotically stable with the $H_{\infty}$ performance constraint (6). In addition, the estimator parameter is computed as $\bar{K}=\bar{P}^{-1} \bar{X}$.

Remark 3: It is well acknowledged that the research on the SE problem for RNNs with time delays has been well investigated. For example, in [24], a sufficient condition has been given to guarantee the exponential stability of the corresponding estimation error dynamics. Nevertheless, most existing estimation schemes are not resistant against the measurement outliers. In other words, once the measurement suffers from abnormal disturbances, traditional state estimators for RNNs are no longer effective.

Remark 4: In Theorems 1 and 2, sufficient criteria have been established for the existence of the desired outlier-resistant state estimator, under which the EED (5) is asymptotically stable with a given $H_{\infty}$ performance level $\hbar$. In Theorem 3 , the gain matrix $K$ is parameterized with the aid of the feasibility of a convex optimization problem. It is observed that the main results obtained in Theorems 1-3 contain all the model information and the performance specifications. The introduction of the saturation function makes the designed estimator capable of weakening the effects from the measurement outliers on the estimation accuracy. .

Remark 5: So far, we have solved the outlier-resistant RSE problem for a class of RNNs subject to mixed time-delays. Compared to existing literature, our main results exhibit two distinctive features: 1) the addressed outlier-resistant state estimation problem is new where a confidence-dependent saturation function is introduced into the estimator structure so as to attenuate the side-effects from the measurement outliers on the EED; and 2) a delay-dependent criterion is derived to guarantee the asymptotic stability of the corresponding EED with guaranteed $H_{\infty}$ performance constraint in the presence of the mixed time-delays.

\section{NUMERICAL EXAMPLE}

In order to demonstrate the effectiveness of our presented RSE scheme on mitigating the effects of measurement outliers, an illustrative example is provided for the delayed RNNs (1) in this section. 
Consider a class of three-neuron RNNs under discrete and distributed time-delays with parameters given as follows:

$$
\begin{aligned}
E & =\operatorname{diag}\{-0.8,-0.6,-0.5\}, \\
D & =\left[\begin{array}{ccc}
-0.3 & 0.2 & 0.3 \\
-0.4 & 0.1 & 0.2 \\
0.2 & -0.4 & 0.2
\end{array}\right], \\
B & =\left[\begin{array}{ccc}
-0.3 & -0.25 & 0.4 \\
0.1 & -0.4 & 0.2 \\
0.6 & 0.2 & -0.3
\end{array}\right], \\
H & =\left[\begin{array}{ccc}
0 & 0.01 & 0 \\
-0.1 & 0.1 & 0 \\
0.2 & 0 & -0.3
\end{array}\right], \\
M & =\left[\begin{array}{ccc}
0.8 & -0.7 & -0.3 \\
0.6 & 0.4 & -0.4
\end{array}\right] .
\end{aligned}
$$

The saturation function $\sigma\left(M e_{k}\right)$ is described as follows:

$$
\sigma\left(M e_{k}\right)=\left\{\begin{aligned}
M e_{k}, & \text { if }-v_{\max } \leq M e_{k} \leq v_{\max }, \\
v_{\max }, & \text { if } M e_{k} \geq v_{\max } \\
-v_{\max }, & \text { if } M e_{k} \leq-v_{\max }
\end{aligned}\right.
$$

where the saturation values are taken as $v_{\max }=1$.

Set the activation functions as

$$
\begin{aligned}
& g\left(x_{k}\right)=\left[\begin{array}{c}
-0.8\left(x_{1 k}\right)+\tanh \left(0.5 x_{1 k}\right)+0.4\left(x_{2 k}\right) \\
0.9\left(x_{2 k}\right)-\tanh \left(0.6 x_{2 k}\right) \\
0.6\left(x_{3 k}\right)-\tanh \left(0.4 x_{3 k}\right)
\end{array}\right], \\
& f\left(x_{k}\right)=\left[\begin{array}{c}
0.2\left(x_{1 k}\right)-\tanh \left(0.1 x_{1 k}\right) \\
0.3\left(x_{2 k}\right)-\tanh \left(0.2 x_{2 k}\right) \\
0.3\left(x_{3 k}\right)-\tanh \left(0.2 x_{3 k}\right)
\end{array}\right] .
\end{aligned}
$$

In this example, according to the engineering practice and with reference to [16], we suppose $\mu_{\tau}=e^{-4 \tau}$, and the discrete timevarying delay $d_{k}$ has the lower bound $\underline{d}=1$ and upper bound $\bar{d}=6$. The disturbance attenuation level is chosen as $\hbar=0.75$. Take the disturbance signals as $w_{k}=\left[\begin{array}{lll}4 e^{-k} & 3 e^{-k} & 5 e^{-k}\end{array}\right]^{T}$. The matrices $L_{1}$, $L_{2}, T_{1}$ and $T_{2}$ in the sector-bounded conditions (3) are given as

$$
\begin{aligned}
& L_{1}=\left[\begin{array}{ccc}
-0.8 & 0.4 & 0 \\
0 & 0.8 & 0 \\
0 & 0 & 0.6
\end{array}\right], \quad L_{2}=\left[\begin{array}{ccc}
-0.3 & 0.4 & 0 \\
0 & 0.2 & 0 \\
0 & 0 & 0.2
\end{array}\right] \text {, } \\
& T_{1}=\left[\begin{array}{ccc}
0.2 & 0 & 0 \\
0 & 0.3 & 0 \\
0 & 0 & 0.3
\end{array}\right], \quad T_{2}=\left[\begin{array}{ccc}
0.1 & 0 & 0 \\
0 & 0.1 & 0 \\
0 & 0 & 0.1
\end{array}\right] \text {. }
\end{aligned}
$$

By solving the LMIs (22) and (27) respectively, the estimator gain matrices are computed as

$$
\begin{aligned}
& K=\left[\begin{array}{cc}
-0.1916 & -0.2144 \\
0.1370 & -0.2649 \\
0.1120 & 0.1970
\end{array}\right], \\
& \bar{K}=\left[\begin{array}{cc}
1.1673 & 1.1673 \\
1.0603 & 1.0603 \\
1.2866 & 1.2866
\end{array}\right] .
\end{aligned}
$$

Now, let us discuss the effectiveness of the outlier-resistant estimator (4) and the traditional estimator (26) under two cases.

Case 1: no measurement outliers occur

If there are no measurement outliers, the evolutions of the estimation errors are shown in Figs. 1-2, where Fig. 1 displays the estimation results utilizing the traditional estimator (26) and Fig. 2 shows the estimation results by using the outlier-resistant estimator (4). The corresponding estimation errors approach zero asymptotically which considerably coincide the theoretical results. The simulation results indicate that both of the two kinds of estimators are effective in estimating the true states when there are no measurement outliers.
Case 2: measurement outliers occur

When the measurement outliers do take place, the simulation results are presented in Figs. 3-5, in which the abnormal disturbances added to $y_{k}$ are characterized in Fig. 3. Obviously, it can be found from Fig. 4 that, the traditional estimator is no longer valid in this case, while the outlier-resistant estimator still works well which can be easily seen from Fig. 5. In summary, the simulation results have verified the necessity of designing the kind of outlier-resistant estimator for the sake of mitigating the effects of measurement outliers on the estimation results.

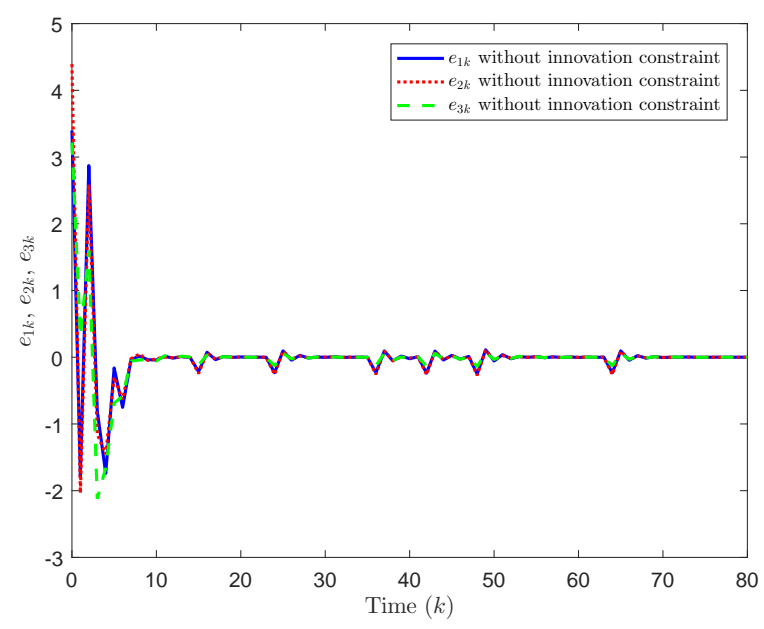

Fig. 1. Estimation errors $e_{1 k}, e_{2 k}$ and $e_{3 k}$ with estimator (26)

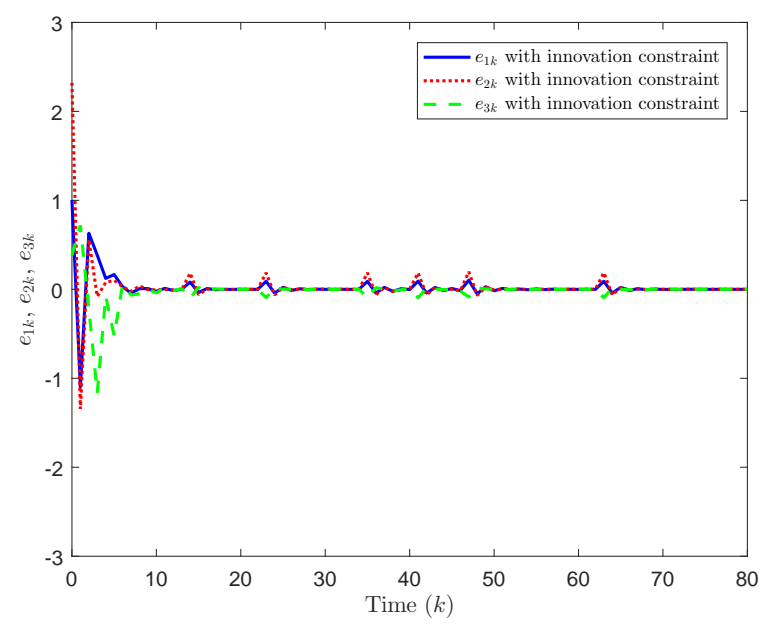

Fig. 2. Estimation errors $e_{1 k}, e_{2 k}$ and $e_{3 k}$ with estimator (4)

\section{Conclusions}

In this paper, considering the appearance of measurement outliers, an outlier-resistant state estimator has been designed for a kind of RNNs subject to mixed time-delays. By introducing a saturation function, an outlier-resistant RSE scheme has been proposed to mitigate the effects of measurement outliers on the estimation results. In order to characterize the actual feature of practical RNNs, the mixed timedelays have been taken into account in current investigation, and a delay-dependent criterion has been derived for the underlying system 


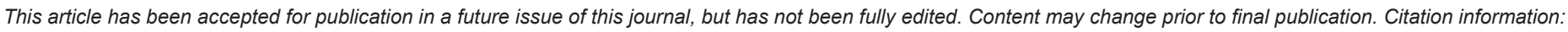
DOI10.1109/TNNLS.2020.2991151, IEEE Transactions on Neural Networks and Learning Systems

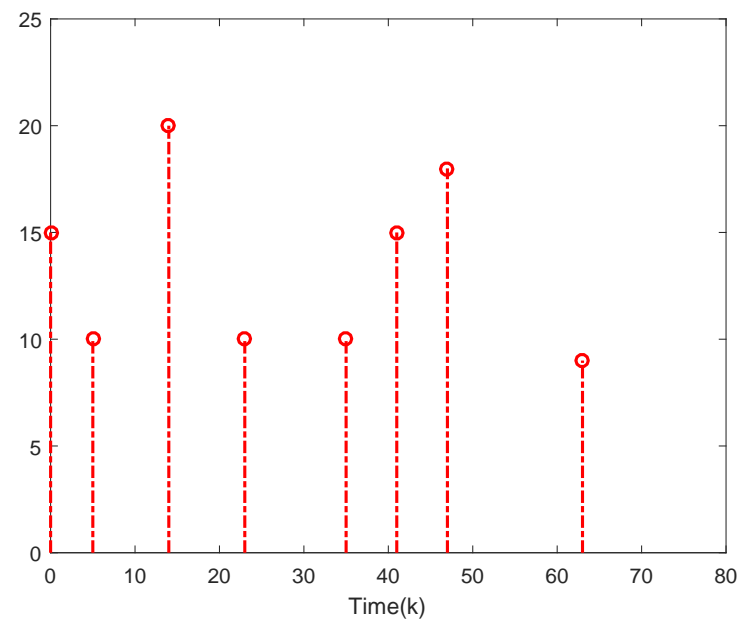

Fig. 3. The abnormal disturbance signals.

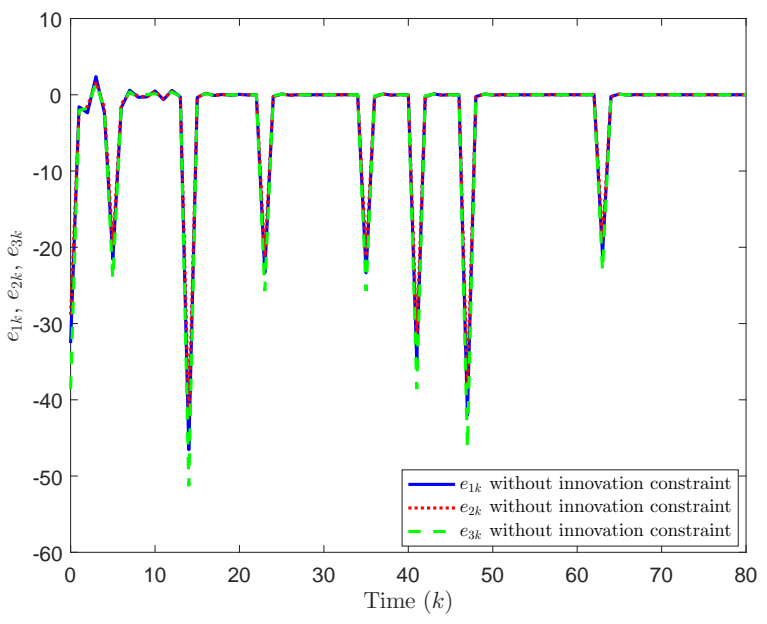

Fig. 4. Estimation errors $e_{1 k}, e_{2 k}$ and $e_{3 k}$ with estimator (26)

(1) to achieve the desired asymptotic stability and $H_{\infty}$ performance constraint. The explicit expression of the estimator gain matrix has been characterized in terms of the solution to a certain LMI. Finally, an illustrative example has been shown to highlight the validity of the outlier-resistant state estimator in estimating the true states of RNNs in the presence of measurement outliers. Besides, one of the future research topics is to extend our proposed outlier-resistant RSE scheme to more complex dynamical networks [13], [14], [52].

\section{REFERENCES}

[1] A. Abdurahman, H. Jiang, and Z. Teng, "Finite-time synchronization for memristor-based neural networks with time-varying delays," Neural Netw., vol. 69, pp. 20-28, 2015.

[2] D. A. Akkaya and M. L. Tiku, "Robust estimation in multiple linear regression model with non-Gaussian noise," Automatica, vol. 44, pp. 407417, 2008.

[3] A. Alessandri and L. Zaccarian, "Stubborn state observers for linear time-invariant systems," Automatica, vol. 88, pp. 1-9, 2018.

[4] G. Battistelli and L. Chisci, "Stability of consensus extended Kalman filter for distributed state estimation," Automatica, vol. 68, pp. 169-178, 2016.

[5] A. Bemporad and A. Garulli, "Output-feedback predictive control of constrained linear systems via set-membership state estimation," Int. J. Control., vol. 73, no. 8, pp. 655-665, 2000.

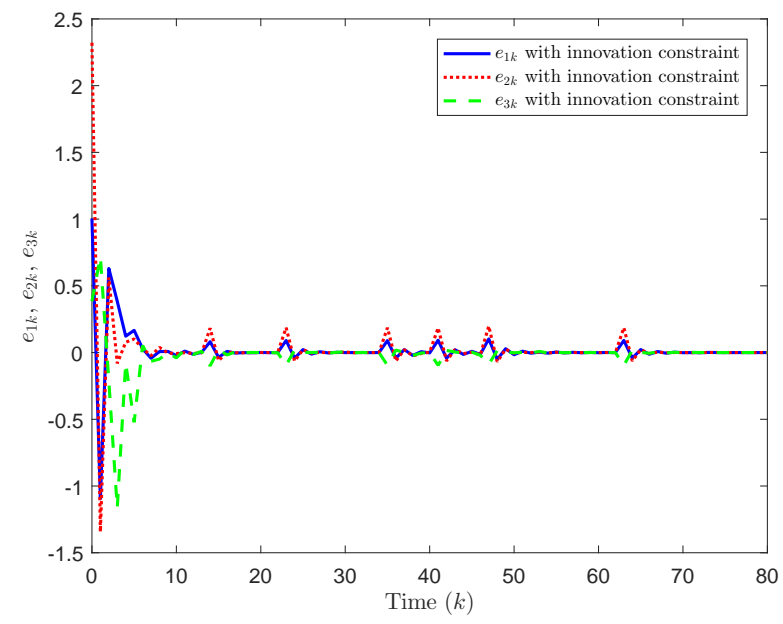

Fig. 5. Estimation error $e_{1 k}, e_{2 k}$ and $e_{3 k}$ with estimator (4)

[6] S. Boyd, L. E. Ghaoui, E. Feron, and V. Balakrishnan, Linear Matrix Inequalities in System and Control Theory, Philadelphia, PA: SIAM, 1994.

[7] J. Cao, Z. Wu, J. Wu, and H. Xiong, "SAIL: Summation-based incremental learning for information-theoretic text clustering," IEEE Trans. Cybern., vol. 43, no. 2, pp. 570-584, 2013.

[8] J. Cao, Z. Bu, Y. Wang, H. Yang, J. Jiang and H.-J. Li, "Detecting prosumer-community group in smart grids from the multiagent perspective," IEEE Transactions on Systems Man Cybernetics-Systems, vol. 49, no. 8, pp. 1652-1664, Aug. 2019.

[9] B. Chen, G. Hu, W. A. Zhang, and L. Yu, "Distributed mixed $H_{2} / H_{\infty}$ fusion estimation with limited communication capacity," IEEE Trans. Autom. Control., vol. 61, no. 3, pp. 805-810, 2016.

[10] X. Chen and Q. Song, "Global stability of complex-valued neural networks with both leakage time delay and discrete time delay on time scales," Neurocomputing, vol. 121, pp. 254-264, 2013.

[11] Y. Chen, Z. Wang, B. Shen and H. Dong, "Exponential synchronization for delayed dynamical networks via intermittent control: Dealing with actuator saturations," IEEE Transactions on Neural Networks and Learning Systems, vol. 30, no. 4, pp. 1000-1012, Apr. 2019.

[12] Y. Chen, Z. Wang, L. Wang and W. Sheng, "Mixed $H_{2} / H_{\infty}$ state estimation for discrete-time switched complex networks with random coupling strengths through redundant channels," IEEE Transactions on Neural Networks and Learning Systems, in press, DOI: 10.1109/TNNLS.2019.2952249.

[13] D. Ding, Z. Wang, Q.-L. Han, and G. Wei, "Security control for a class of discrete-time stochastic nonlinear systems subject to deception attacks," IEEE Trans. Syst., Man, Cybern., Syst., vol. 48, no. 5, pp. 779-789, 2018.

[14] H. Dong, Z. Wang, and H. Gao, "Fault detection for Markovian jump systems with sensor saturations and randomly varying nonlinearities," IEEE Trans. Circuits Syst. I, Reg. Papers, vol. 59, no. 10, pp. 2354 2362, 2012.

[15] M. A. Gandhi and L. Mili, "Robust Kalman filter based on a generalized maximum-likelihood-type estimator," IEEE Trans. Signal Process., vol. 58 , pp. 2509-2520, 2010.

[16] K. Gu, J. Chen, and V L. Kharitonov, Stability of time-delay systems, Springer Science \& Business Media, 2003.

[17] F. Han, Z. Wang, H. Dong, and H. Liu, "Partial-nodes-based scalable Hconsensus filtering with censored measurements over sensor networks," IEEE Trans. Syst., Man, Cybern., Syst., in press, DOI: 10.1109/TSMC.2019.2907649.

[18] M. Hernandez-Gonzalez, E. A. Hernandez-Vargas, and M. V. Basin, "Discrete-time high order neural network identifier trained with cubature Kalman filter," Neurocomputing, vol. 322, pp. 13-21, 2018.

[19] N. Hou, H. Dong, Z. Wang, W. Ren, and F. E. Alsaadi, " $H_{\infty}$ state estimation for discrete-time neural networks with distributed delays and randomly occurring uncertainties through fading channels," Neural Netw., vol. 89, pp. 61-73, 2017.

[20] H. Huang, T. Huang, and X. Chen, "Further result on guaranteed $H_{\infty}$ performance state estimation of delayed static neural networks," IEEE Trans. Neural Netw. Learn. Syst.," vol. 26, no. 6, pp. 1335-1341, 2015. 
[21] G. Joya, M. A. Atencia, and F. Sandoval, "Hopfield neural networks for optimization: Study of the different dynamics," Neurocomputing, vol. 43, no. 1-4, pp. 219-237, 2002.

[22] H. R. Karimi and H. Gao,"New delay-dependent exponential $H_{\infty}$ synchronization for uncertain neural networks with mixed time delays," IEEE Trans. Syst., Man, Cybern. B, Cybern., vol. 40, no. 1, pp. 173-185, 2009.

[23] J. Li, H. Dong, Z. Wang, and X. Bu,"Partial-neurons-based passivityguaranteed state estimation for neural networks with randomly occurring time-delays," IEEE Trans. Neural Netw. Learn. Syst., in press, DOI:10.1109/TNNLS.2019.2944552.

[24] T. Li and S. M. Fei, "Exponential state estimation for recurrent neural networks with distributed delays," Neurocomputing, vol. 71, nos. 1-3, pp. 428-438, 2007.

[25] M. Liu and $\mathrm{H}$. Chen, " $H_{\infty}$ state estimation for discrete-time delayed systems of the neural network type with multiple missing measurements," IEEE Trans. Neural Netw. Learn. Syst., vol. 26, no. 12, pp. 2987-2998, 2015.

[26] S. Liu, G. Wei, Y. Song, and Y. Liu, "Extended Kalman filtering for stochastic nonlinear systems with randomly occurring cyber attacks," Neurocomputing, vol. 207, pp. 708-716, 2016.

[27] Y. Liu, B. Shen, and H. Shu, "Finite-time resilient $H_{\infty}$ state estimation for discrete-time delayed neural networks under dynamic event-triggered mechanism," Neural Netw., vol. 121, pp. 356-365, 2020.

[28] Y. Liu, Z. Wang, Y. Yuan and W. Liu, "Event-triggered partial-nodesbased state estimation for delayed complex networks with bounded distributed delays," IEEE Transactions on Systems, Man, and CyberneticsSystems, vol. 49, no. 6, pp. 1088-1098, Jun. 2019.

[29] Y. Liu, Z. Wang, and D. H. Zhou, "UKF-based remote state estimation for discrete artificial neural networks with communication bandwidth constraints," Neural Netw., vol. 108, pp. 393-398, 2018.

[30] Y. Luo, Z. Wang, G. Wei, F. E. Alsaadi, and T. Hayat, "State estimation for a class of artificial neural networks with stochastically corrupted measurements under Round-Robin protocol," Neural Netw., vol. 77, pp. 70-79, 2016.

[31] Y. Nakahira and Y. Mo, "Attack-resilient $\mathcal{H}_{2}, \mathcal{H}_{\infty}$, and $\ell_{1}$ state estimator," IEEE Trans. Autom. Control., vol. 63, no. 12, pp. 4353-4360, 2018.

[32] Y. V. Pershin and M. Di Ventra, "Experimental demonstration of associative memory with memristive neural networks," Neural Netw. vol. 23, no. 7, pp. 881-886, 2010.

[33] R. Sakthivel, P. Vadivel, K. Mathiyalagan, A. Arunkumar, and M. Sivachitra, "Design of state estimator for bidirectional associative memory neural networks with leakage delays," Inf. Sci., vol. 296, pp. 263-274, 2015.

[34] A. V. Savkin and I. R. Petersen, "Set-valued state estimation via a limited capacity communication channel," IEEE Trans. Autom. Control., vol. 48, no. 4, pp. 676-680, 2003.

[35] N. Sengupta, M. Sahidullah, and G. Saha, "Lung sound classification using cepstral-based statistical features," Comput. Biol. Med., vol. 75, pp. 118-129, 2016.

[36] B. Shen, Z. Wang, and H. Tan, "Guaranteed cost control for uncertain nonlinear systems with mixed time-delays: the discrete-time case," Eur. J. Control, vol. 40, pp. 62-67, 2018.

[37] H. Shen, Y. Zhu, L. Zhang, and J. H. Park, "Extended dissipative state estimation for Markov jump neural networks with unreliable links," IEEE Trans. Neural Netw. Learn. Syst., vol. 28, no. 2, pp. 346-358, 2017.

[38] K. Suzuki, H. Abe, H. MacMahon, and K. Doi, "Image-processing technique for suppressing ribs in chest radiographs by means of massive training artificial neural network (MTANN)," IEEE Trans. Med. Imaging, vol. 25 , no. 4 , pp. 406-416, 2006.

[39] X. Wan, Z. Wang, M. Wu and X. Liu, " $H_{\infty}$ state estimation for discretetime nonlinear singularly perturbed complex networks under the RoundRobin protocol," IEEE Transactions on Neural Networks and Learning Systems, vol. 30, no. 2, pp. 415-426, Feb. 2019.

[40] Y. Wang, Y. Xia, P. Zhou, and D. Duan, "A new result on $H_{\infty}$ state estimation of delayed static neural networks," IEEE Trans. Neural Netw. Learn. Syst., vol. 28, no. 12, pp. 3096-3101, 2016.

[41] Z. Wang, Y. Xu, R. Lu, and H. Peng, "Finite-time state estimation for coupled Markovian neural networks with sensor nonlinearities," IEEE Trans. Neural Netw. Learn. Syst., vol. 28, no. 3, pp. 630-638, 2015.

[42] G. Wei, S. Liu, Y. Song, and Y. Liu, "Probability-guaranteed setmembership filtering for systems with incomplete measurements," $\mathrm{Au}$ tomatica, vol. 60, pp. 12-16, 2015.

[43] Y. Xu, R. Lu, P. Shi, J. Tao, and S. Xie, "Robust estimation for neural networks with randomly occurring distributed delays and Markovian jump coupling," IEEE Trans. Neural Netw. Learn. Syst., vol. 29, no. 4 pp. $845-855,2018$.
[44] H. Yan, F. Qian, F. Yang, and H. Shi, " $H_{\infty}$ filtering for nonlinear networked systems with randomly occurring distributed delays, missing measurements and sensor saturation," Inf. Sci., vol. 370, pp. 772-782, 2016.

[45] F. Yang and Y. Li, "Set-membership filtering for systems with sensor saturation," Automatica, vol. 45, no. 8, pp. 1896-1902, 2009.

[46] X. Yang, J. Cao, and J. Lu, "Synchronization of randomly coupled neural networks with Markovian jumping and time-delay," IEEE Trans. Circuits Syst. I, Reg. Papers, vol. 60, no. 2, pp. 363-376, 2013.

[47] X. Yang and D. W. C. Ho, "Synchronization of delayed memristive neural networks: robust analysis approach," IEEE Trans. Cybern, vol. 46, no. 12 , pp. 3377-3387, 2016.

[48] X.-M. Zhang and Q.-L. Han, "State estimation for static neural networks with time-varying delays based on an improved reciprocally convex inequality," IEEE Trans. Neural Netw. Learn. Syst., vol. 29, no. 4, pp. 1376-1381, 2017

[49] X.-M. Zhang, Q.-L. Han, and X. Ge, "An overview of neuronal state estimation of neural networks with time-varying delays," Inf. Sci. vol. 478, pp. 83-99, 2019.

[50] D. Zhao, Z. Wang, Y. Chen and G. Wei, "Proportional-integral observer design for multi-delayed sensor-saturated recurrent neural networks: A dynamic event-triggered protocol," IEEE Transactions on Cybernetics, in press, $10.1109 /$ TCYB.2020.2969377

[51] B. Zhou and Q. Song, "Boundedness and complete stability of complexvalued neural networks with time delay," IEEE Trans. Neural Netw. Learn. Syst., vol. 24, no. 8, pp. 1227-1238, 2013.

[52] L. Zou, Z. Wang, J. Hu and D. Zhou, "Moving horizon estimation with unknown inputs under dynamic quantization effects", IEEE Trans. Autom. Control., in press, DOI: 10.1109/TAC.2020.2968975. 